\title{
La prise en charge par l'hôpital des populations à la marge du système de santé en France : l'exemple des Permanences d'accès aux soins de santé
}

The government of groups at the margin of the healthcare system in French public hospital: the example of the department for access to healthcare

\section{Jérémy Geeraert}

Institut de recherche interdisciplinaire sur les enjeux sociaux. Paris, France.

E-mail : geeraert.jळgmail.com

\section{Courrier}

Brunnenstr., 6. Berlin, Allemagne. 10119

\section{Résumé}

Cet article analyse la prise en charge de la pauvreté à l'hôpital public. Il prend l'exemple des Permanences d'accès aux soins de santé (PASS), structure hospitalière qui prend en charge des personnes connaissant des difficultés d'accès aux soins dans le système de santé français. Il s'appuie sur une enquête empirique issue d'un travail de thèse en sociologie. L'enquête mêle observations participantes et entretiens semi-directifs $(n=49)$ et a été réalisée dans 16 PASS différentes. Sur un plan théorique, l'article met en miroir les travaux sur les métamorphoses de la question sociale et une perspective biopolitique d'inspiration foucaldienne. La prise en charge des populations en marge du système de santé par une institution publique (l'hôpital) est considérée comme agissant dans des domaines qui dépassent le strict traitement de la maladie pour entrer dans ceux de la régulation sociale, la protection de la santé publique ou la réalisation de droits humains. Dans le cas des PASS, l'article montre la construction d'une biopolitique de la migration autour d'un bricolage compliqué qui mêle citoyenneté, politiques économiques, politiques de santé publique et accès aux soins. Le mode d'exercice du pouvoir se caractérise par un gouvernement par le bas qui est réalisé par les professionnels de terrain lors des interactions avec les patients et met en scène une économie morale basée sur des représentations de ce qui est bon et mauvais pour la société.

Mots-clés : Accès aux Soins; Hôpital Public; Question Sociale; Biopolitique; Migration. 
This article analyses how the modern public hospital handles poverty. To do so it goes from the example of the hospital-based Departments for access to healthcare (Permanences d'accès aux soins de santé - PASS), that is specialized in taking care of patients experiencing difficulties to access healthcare in the French healthcare system. The article relies on an empirical study achieved as part of a PhD work in sociology. The study combines participant observations and interviews $(n=49)$ which have been conducted in 16 different PASS. On a theoretical level, the paper mirrors the work on the metamorphosis of the social question and a biopolitics perspective inspired by Foucault. In this perspective the way the public hospital takes care of individuals situated at the margin of the healthcare system is thought as entering in the areas of social regulation, protection of the public health or realization of human right. In the case of PASS, the article shows the construction of a biopolicy of migration that is a cobbling together of citizenship, economic policies, public health and access to healthcare. The form of power is characterized by a government from the bottom which is realized by street-level healthcare professionals during interaction with the patients and based on a moral economy depicting representations of what is good and bad for the society.

Keywords: Access to Healthcare; Public Hospital; Social Question; Biopolitics; Migration.
L'hôpital public en occident a incarné jusqu'au début du $\mathrm{xx}^{\mathrm{e}}$ siècle une mission d'assistance et un principe de charité institutionnelle. ${ }^{1}$ Il avait cependant peu de fonctions soignantes. C'était un lieu d'accueil pour les déshérités, les personnes âgées et malades où l'on ne venait bien souvent que pour y mourir (Foucault et al., 1979). Robert Castel situe dans le Haut Moyen-Âge la naissance de ce qu'il appelle le «social-assistanciel » prenant place dans une société qui se complexifie et où les liens sociaux se distendent. La prise en charge des démunis va alors faire l'objet de pratiques spécialisées : orphelinat, hôpitaux, distribution organisée de l'aumône (Castel, 2007). Ces dernières participent au maintien de la cohésion et de la stabilité d'une société en ce qu'elles visent à prendre en charge les individus exclus de la société ou menacés de l'être. Il voit dans la naissance et le développement du social-assistanciel, la solution que les sociétés complexes proposent pour résoudre les problèmes internes qu'elles rencontrent en matière de protection et d'intégration des catégories menacées de désaffiliation. ${ }^{2}$

La fonction d'assistance de l'hôpital est à articuler avec une autre fonction, celle du contrôle social et d'enfermement des pauvres qui s'accentue fortement partout en Europe à partir du xvii siècle en raison d'une forte augmentation de la pauvreté (Foucault, 1976b; Geremek 2010). Les hôpitaux généraux, créés en France à la fin du XVIII ${ }^{\mathrm{e}}$ siècle, incarnent ce tournant; ils oscillent alors entre un rôle correctionnel et un caractère carcéral (Fossier, 2002), tout en maintenant une fonction de secours des indigents. Lorsqu'on le considère en dehors (ou au-delà) de sa fonction soignante, l'hôpital se caractérise donc par cette ambivalence entre secours d'un côté et répression et contrôle social de l'autre.

Avec la médicalisation de l'hôpital à partir du XIXe siècle, l'institution se détache peu à peu de ses fonctions d'assistance et de répression

\footnotetext{
1 Pour ne citer que quelques ouvrages majeurs : Imbert (1966), Imbert et Mollat (1982) et Geremek (2010). Pour une analyse sociologique, voir Castel (2007), Foucault et al. (1979) et Foucault (2009).

2 R. Castel définit la désaffiliation comme la dissociation du lien social en raison de l'absence de travail et de l'isolement social. C'est « le décrochage par rapport aux régulations à travers lesquelles la vie sociale se reproduit et se reconduit » (Castel, 2009, p. 92).
} 
pour devenir de plus en plus exclusivement le lieu du soin technique et spécialisé que nous connaissons aujourd'hui. Elle s'inscrit dans la «biopolitique » naissante identifiée par Michel Foucault et caractérisée par une transformation de la forme du pouvoir étatique qui s'oriente de plus en plus vers l'entretien et le gouvernement de la vie des citoyens (Foucault, 1976a, 2004). L'hôpital devient ainsi peu à peu le lieu du contrôle des corps et des comportements. La prise en charge des populations démunies dans ce contexte n'est plus seulement comprise comme un acte de charité ou un devoir moral, mais également (et surtout) comme un programme biopolitique en vue de faire prospérer la nation dans un contexte de concurrence internationale. Cette dimension est une caractéristique essentielle des politiques de santé orientées vers les catégories les moins privilégiées qui trouve encore une forte résonance aujourd'hui (Borgetto, 2013).

Comment étudier dans une perspective sociologique la prise en charge de la pauvreté dans l'espace hospitalier de nos sociétés modernes? Cette question qui servira de fil rouge à cet article, permettra de poser un état de la recherche française dans ce domaine et de présenter quelques résultats d'une recherche menée par l'auteur sur ce sujet. Sur un plan théorique, l'article met en miroir les travaux sur les métamorphoses de la question sociale réalisés par Robert Castel et une perspective biopolitique dans la lignée des travaux d'inspiration foucaldienne. Il s'agit de considérer la prise en charge des populations en marge du système de santé par une institution publique (l'hôpital) comme agissant dans des domaines qui dépassent le strict traitement de la maladie pour entrer dans ceux de la régulation sociale, la protection de la santé publique ou la réalisation de droits humains. Sur un plan empirique, cet article s'appuie sur une enquête réalisée dans le cadre d'une thèse en sociologie sur les Permanences d'accès aux soins de santé (PASS). ${ }^{3}$ Créées en 1998 dans le cadre de la loi de lutte contre les exclusions, les PASS sont des structures hospitalières assistancielles qui offrent soins et médicaments aux patients connaissant des difficultés d'accès aux soins dans le système de santé (par exemple en raison d'une absence de couverture maladie). Elles n'ont pas de fonction d'hébergement, mais elles poursuivent une mission d'assistance aux plus démunis (Geeraert, 2017b).

Pour cela nous verrons dans une première partie les caractéristiques qui façonnent le cadre de la prise en charge des personnes les plus démunis à l'hôpital public moderne, notamment celles du système de protection maladie et celles des représentations sociales sur la définition de la santé. Dans un second temps, nous verrons plus précisément comment la sociologie peut étudier la prise en charge à l'hôpital des personnes menacées d'exclusion sociale en prenant pour exemple la PASS. Cela nous amènera à interroger d'une manière transversale les apports heuristiques d'une approche croisant le thème de la biopolitique avec celui du traitement de la question sociale.

\section{Une nouvelle définition de la santé}

$\mathrm{Au}$ cours du $\mathrm{XX}^{\mathrm{e}}$ siècle, et surtout à partir de sa seconde moitié, les sociétés occidentales ont construit des systèmes de protection sociale tendant à couvrir l'ensemble de la population contre des risques sociaux (maladie, vieillesse, chômage). La construction d'un État social qui protège la population face aux grands risques sociaux s'ajoute et dépasse le système traditionnel du social-assistanciel évoqué dans l'introduction et agit comme un fort régulateur de la société visant l'intégration du plus grand nombre. Castel voit dans le développement d'un État social fort une tentative pour maintenir la cohésion sociale face aux risques de fractures sociales (intrinsèques à toutes les sociétés, mais augmentés dans le modèle capitaliste en raison des fortes inégalités sociales qu'il produit). La société agit alors sur elle-même afin de reconstruire de la cohésion sociale là où elle est la plus faible (Castel, 2007).

\footnotetext{
3 L'enquête a été conduite entre 2011 et 2014 dans 16 PASS différentes réparties sur le territoire français. Lors de celle-ci des observations ont été réalisées dans les salles d'attente, lors des consultations médicales et des entrevues avec le travailleur social. 42 entretiens semidirectifs ont été réalisés, principalement auprès des professionnels des PASS (médecins, travailleurs sociaux, personnel infirmier).
} 
Au-delà d'un moyen de réguler la société, la protection de la santé s'érige en droit. La France entérine à partir de 1946 un véritable « droit au soin » qui fait écho à la nouvelle définition de la santé portée dans le monde occidental et qu'on peut résumer par « un état de complet bien-être physique, mental et social », et ne consiste pas seulement «en une absence de maladie ou d'infirmité » (OMS, 1946, p. 16). Plus important encore pour notre propos, ce nouveau droit au soin est le fruit d'un combat social qui tend à protéger la vie elle-même et reconnaît chez tous les individus une "bio-légitimité », c'està-dire une « reconnaissance supérieure donnée au principe de maintien en vie » (Fassin; Costa-Lascoux; Hily, 2001, p. 146). Ce droit dépasse le seul calcul biopolitique évoqué dans l'introduction pour entrer dans le champ de la morale.

Pour résumer, on peut dire que la conception de la santé moderne comporte trois caractéristiques majeures. 1. Elle est paradigmatique et totalisante (Gori; Del Volgo, 2009) ; tendant à gouverner les corps et les comportements (Fassin; Memmi, 2004), notamment pour discipliner la société et la rendre plus productive. 2. Elle vise à défendre la vie, érigée en valeur fondamentale (bio-légitimité), dans une perspective de droit de l'homme et de justice sociale. 3. Elle est constituée comme un risque social pouvant mener, lorsqu'elle n'est pas ou mal protégée, à un défaut d'intégration social.

\section{Les effets du contexte}

\section{La maîtrise de l'augmentation des dépenses de santé}

Tout en même temps, la généralisation de la protection de la santé à tous les travailleurs et à leur famille, la modernisation du système de santé et les évolutions démographiques (notamment le vieillissement de la population) et culturelles (augmentation des besoins de santé et médicalisation croissante de nouveaux domaines de la vie) ont conduit à une augmentation sans précédent des dépenses de santé dans la société française d'aprèsguerre (Gori; Del Volgo, 2009; Sicard; Le Pen, 2004). Ces dépenses de santé sont ainsi passées de 2,6\% en 1950 à 9,1 \% du PIB en 2010 (Le Garrec; Koubi;
Fenina, 2013). Cette croissance structurelle n'est pas propre à la France. Elle se retrouve aussi chez nos voisins européens (Colombo; Morgan, 2006). Les politiques visant à maîtriser cette augmentation se sont accentuées depuis les années 1970. Le sociologue Frédéric Pierru a montré les grandes tendances de ces réformes : refus d'augmenter les recettes par une hausse conséquente des prélèvements obligatoires, privatisation et déremboursement de l'assurance maladie, baisse du taux de prise en charge publique (Pierru, 2007). Face aux puissants groupes de pression que constituent les médecins d'un côté, les assurances privées et l'industrie pharmaceutique de l'autre, la marge de manœuvre des politiques de santé pour redresser les comptes de l'Assurance maladie a été réduite et les tentatives de réforme du système de santé ont souvent été bloquées. D'une manière générale, les économies réalisées ont largement été portées par les consommateurs et justifiées par des discours de responsabilisation des comportements individuels. Selon F. Pierru, cette volonté de responsabiliser les individus dans leur consommation de soin est inefficace et conduit à augmenter les inégalités sociales de santé car ce sont les personnes les plus pauvres qui ont le plus de mal à couvrir les coûts laissés alors à leur charge. Ainsi, les politiques de maîtrise de l'augmentation des dépenses de santé ont conduit à faire porter une partie toujours plus importante des coûts aux particuliers, défavorisant les groupes les moins dotés économiquement, et particulièrement ceux qui n'entrent pas dans le système de couverture maladie. Si cette tendance est générale au système de santé dans son ensemble, elle se retrouve en particulier à l'hôpital.

Les réformes hospitalières de ces trente dernières années, qui se sont accompagnées d'un resserrement de plus en plus important des budgets face à une croissance de l'activité plutôt constante, ont entraîné des restrictions budgétaires et contraint à augmenter la productivité et à faire des économies qui n'ont pu être que partiellement compensées par les réorganisations (Belorgey, 2010; Domin, 2007; Pierru, 2007). L'hôpital public moderne, depuis le début de la crise économique au milieu des années 1970, se caractérise de plus en plus par sa dimension économique. Les budgets quasiment illimités, dont disposaient les hôpitaux d'avant la Crise, sont remis 
en question. C'est tout d'abord le modèle économique de «l'hôpital-entreprise » développé à la fin des années 1970 qui a légitimé scientifiquement ce tournant (voir Benamouzig, 2005; Domin, 2007). Frédéric Pierru a montré que ces réformes ont amené l'instauration d'un fonctionnement libéral et gestionnaire.

Une partie des réformes hospitalières s'inspirent du New Public Management (NPM) agissant principalement sur l'organisation de l'activité. Ce courant de pensée n'est pas circonscrit à l'espace de l'hôpital, au contraire, il s'est largement imposé dans la plupart des bureaucraties des sociétés occidentales. Il se concrétise dans la « nouvelle gestion publique » qui se retrouve dans la gestion de presque toutes les administrations depuis les années 1990, avec une forte accélération dans les années 2000 (Bezes, 2012). Le NPM est une nouvelle manière de penser les systèmes administratifs publics. Il consiste en des techniques de gestions qui s'inspirent des méthodes de management de l'entreprise privée.

Le sociologue Nicolas Belorgey dit à ce propos :

[Le NPM] s'intéresse au contrôle des agents producteurs, cherchant à accroitre leur productivité et leur fixant à cette fin des objectifs quantifiés. Ses outils de motivation sont alors essentiellement financiers. À son prisme, les questions sont reformulées en termes purement techniques, les aspects politiques étant réputés traités dans des instances extérieures comme le Parlement. Il se présente comme une simple recherche d'efficacité, donc transposable dans n'importe quel contexte, au service de n'importe quelle fin. Sous ce jour, il n'y aurait pas de différence fondamentale entre le secteur privé et public, et il serait donc temps d'importer dans le second qui serait si inefficace et irait si mal, les outils qui auraient fait la fortune du premier. (Belorgey, 2010, p. 9)

Le travail de Nicolas Belorgey sur l'arrivée du new public management (NPM) à l'hôpital public au travers des réformes construisant la nouvelle gouvernance hospitalière laisse entrevoir l'accroissement des inégalités d'accès aux soins et aux services de santé provoqué par la standardisation des pratiques médicales aux dépens des patients les moins bien dotés socialement et économiquement. Il montre néanmoins que ces réformes ne s'appliquent pas de manière uniforme et qu'elles font l'objet de résistance de la part de nombreux professionnels (Belorgey, 2010).

D'autres travaux ont étudié les effets du tournant néo-managérial dans les administrations de l'État social remplissant des missions auprès de publics précaires (Gabarro, 2012; Martin, 2016; Siblot, 2006). Les phénomènes de «dualisation » des services publics qui se divisent entre des dispositifs destinés à l'ensemble de la population «suivant des normes d'efficacité gestionnaire » et des services spécifiques réservés aux plus démunis entrainent des représentations culturalistes des publics les plus démunis. Ces derniers sont compris comme « difficiles », voire « agressifs » (Siblot, 2005, p. 71) renforçant leur stigmatisation et permettent « un désengagement discret des institutions publiques au nom même de l'amélioration du service aux populations “en difficulté” »(Siblot, 2005, p. 87). La course à la productivité et à la rationalisation tend par ailleurs à cantonner les personnes aux situations complexes dans des circuits spécifiques sous-dotés et embouteillés limitant leur accès aux droits et aux prestations (Gabarro, 2012; Martin, 2016).

\section{Généralisation et stratification des systèmes de protection maladie}

Au niveau des systèmes de couverture maladie, on note en France une tendance à leur généralisation à l'ensemble de la population à partir de la fin de la Seconde guerre mondiale. L'arrivée à la fin des années 1990 de la Couverture maladie universelle complémentaire (protégeant la santé des personnes pauvres et en situation régulière) et de l'Aide médicale d'État (protégeant la santé des personnes pauvres et en situation irrégulière) complètent ce processus de protection de la santé pour l'ensemble de la population. Des dispositifs d'assistance, tel que la PASS, offrent un accès aux soins limités et ponctuels à ceux qui échapperaient aux systèmes de couverture maladie.

Parallèlement à ce phénomène, on assiste sur la même période à la construction inédite (grâce 
à des outils juridiques et à la stigmatisation) d'un groupe social - les étrangers - dont l'accès au système de santé a été bridé pour certaines de ses franges (Carde, 2009; Izambert, 2010; Geeraert, 2017 b). Les instruments de protection maladie se démultiplient et se réduisent à mesure que la situation administrative de séjour des personnes se détériore. La stratification fine des statuts de séjour qui s'est développée depuis les années 1970 en France s'accompagne d'une différenciation des droits en matière de protection de la santé.

Il faut noter que la France fait figure d'exception dans ce domaine et est la seule parmi ses voisins européens à offrir une couverture maladie fonctionnelle et aussi large à ses ressortissants irréguliers. C'est le paradoxe du système de protection maladie français qui intègre et protège des franges de la population qu'il a isolées et stigmatisées en amont et qui, en même temps, exclut certains groupes parmi les plus précaires à l'intérieur de cette frange. Les exclus parmi les étrangers précaires sont ceux qu'on retrouvera dans les PASS qui constitue l'un des derniers filets de protection de la santé. Ainsi, la grande majorité des patients des PASS ont deux points communs qui montrent que leur place dans le système de santé est directement liée à des caractéristiques socialement construites. En plus de faire l'expérience d'une précarité économique et sociale, les patients des PASS sont, la plupart du temps, étrangers au statut de résidence précaire. On retrouve ici la binarité entre inclusion et exclusion évoquée dans l'introduction. Inexorablement, tout se passe comme si le développement de l'État social sur la période récente produit, en contrepartie de l'inclusion de plus en plus large de la population dans le système de l'assurance maladie, une désaffiliation d'autant plus grande de ses marges.

La question de l'accès aux soins dans la France contemporaine doit être comprise comme intimement liée à la question de la citoyenneté qui est devenue un instrument de domination par le maintien dans la précarité dans de nombreux domaines de l'existence de certaines catégories d'étrangers. À contre-courant du mouvement de généralisation de la protection maladie, l'isolement et l'exclusion de certains groupes d'étrangers stigmatisés tracent les traits d'un système de santé stratifié à ses marges s'appuyant sur des statuts de citoyenneté. À cet égard, la protection de la santé, au travers de l'accès aux soins et au système de santé, devient un instrument biopolitique participant à la formation et à la reproduction d'un ordre social stratifié.

\section{La fonction sociale à l'hôpital}

La réforme de 1958 de Robert Debré ${ }^{4}$ confirme le visage de l'hôpital moderne français comme celui d'un temple de la technique. Elle crée le temps plein hospitalier et le centre hospitalo-universitaire qui attire les grands spécialistes et accapare les moyens techniques modernes. Il assure une triple fonction : le soin, l'enseignement et la recherche (Jamous, 1969). Cette période s'accompagne d'un spectaculaire accroissement des dépenses de santé dû au progrès technique, mais aussi à l'élargissement de la clientèle et des besoins et demandes de soins (Steudler, 1973, p. 34). Les sociologues écrivant sur l'hôpital au début des années 1970 observent un recentrage de l'activité sur les pathologies ayant un intérêt scientifique, alors que les maladies ordinaires sont traitées par la médecine de ville. La mission traditionnelle d'assistance " paraît s'opérer au détriment d'une vocation plus moderne, autre que celle de l'asile, [qui] en vient à être dévalorisée par l'hôpital lui-même comme par le corps médical » (Herzlich, 1973, p. 46). Le développement de l'activité hospitalière semble s'accompagner inexorablement d'une spécialisation des services et de la mise à l'écart de la médecine de premier recours (Chauvenet, 1973; Vassy; Renard, 1992). Les services sont de plus en plus spécialisés, sélectifs (dans le recrutement des patients qui se fait selon leur pathologie) et agissent à une échelle déterritorialisée.

La fonction asilaire historique a aujourd'hui quasiment disparue de l'hôpital public. Elle s'est exportée dans d'autres établissements, comme les

4 Décret $n^{0} 5$ 8-1202 du 11 décembre 1958 relatif aux hôpitaux et hospices publics. 
établissements d'hébergement pour personnes âgées dépendantes, par exemple. Cependant, l'hôpital public continue de remplir une mission «sociale » qui ne peut se dissoudre complètement dans une activité purement médicale ou technique. La création de la fonction d'assistante sociale hospitalière à partir de 1945 est le signe du maintien de cette dimension. Les permanences d'accès aux soins (PASS) en sont un autre exemple.

Nicolas Dodier et Agnès Camus $(1997,1998)$ ont souligné la permanence de cette dualité de l'hôpital public contemporain qui se caractérise par une «tension entre ouverture et fermeture ». La fermeture désigne le processus de spécialisation et de sélection des patients que nous venons de décrire. L'ouverture, quant à elle, s'incarne au travers de la notion de service public hospitalier institué par la loi de $1970^{5}$ et de ses valeurs - l'égalité de l'accès, l'égalité de traitement et la continuité du service. Plus concrètement, on la retrouve dans les services ouverts (médecine interne, service de médecine générale, urgences) qui s'inscrivent dans un territoire avec des patients recrutés localement. Le lieu le plus ouvert est le service des urgences. Plusieurs autres espaces symbolisent également cette ouverture, au premier rang desquels la PASS ou les services de gériatrie et de médecine palliative et quelques autres services qui se sont développés depuis la seconde moitié du $\mathrm{xx}^{\mathrm{e}}$ siècle comme par exemple les pôles de santé publique (médecine préventive, centres d'interruption volontaire de grossesse, de dépistage etc.), la médecine carcérale ou les consultations pour patients sourds. Ces services continuent de recevoir des patients parmi les classes sociales les moins favorisées. Les pôles de santé publique soulignent l'importance des missions biopolitiques de l'hôpital public. Ces dernières sont à la fois spécialement dirigées vers les patients les plus démunis pour garantir le respect du principe de bio-légitimité et visent à protéger la santé de la population dans son ensemble (notamment dans le dépistage et la prise en charge des maladies infectieuses et contagieuses).

Le service des urgences, étudié par Dodier et Camus (1997), illustre bien cette dualité entre ouverture et fermeture. Alors qu'il est ouvert à tous et tout le temps, la sélection des patients dans un second temps écarte les demandes qui ne sont pas de "vraies urgences " (dont notamment les demandes dites «sociales ", p. 114) de celles vitales ou nécessitant une prise en charge médicale immédiate. Cette tendance à la sélection des patients, caractéristique de la fermeture, génère de la part des professionnels de santé une «mobilisation différenciée » (p. 113) selon les patients dont la conséquence peut aller de la réorientation du patient à une place reculée dans l'ordre de passage. Dans ce contexte, les patients détiennent une «valeur mobilisatrice » plus ou moins importante (p. 113) qui se corrèlent à leur statut social qui va déterminer leurs chances d'accès aux soins.

En contrepartie, les auteurs soulignent que la notion de «service public » est une valeur importante pour de nombreux professionnels du soin travaillant à l'hôpital, elle s'exprime dans une volonté d'ouvrir l'hôpital à tous ceux qui s'y présentent, et en particulier aux catégories sociales défavorisées n'ayant pas la possibilité de se rendre dans d'autres établissements de soin. Cette valeur est renforcée par l'éthique médicale dont le serment d'Hippocrate est une référence forte. L'hôpital public porte ainsi une médecine sociale et éthique qui est présente en son sein d'une manière diffuse.

La prise en charge des publics en marge du système de santé par l'hôpital public français moderne est façonnée par trois phénomènes principaux : (1) la tendance vers la spécialisation et la technicisation du soin ; (2) l'évolution de l'organisation budgétaire, de plus en plus contrainte et de plus en plus orientée vers une logique de rentabilité ; (3) l'exclusion des systèmes de couverture maladie principaux de certains groupes précarisés parmi les étrangers. Les deux premiers phénomènes conduisent à réduire la fonction sociale de l'hôpital et tendent à écarter les patients les plus démunis des circuits de soins. Ces derniers sont de plus en plus cantonnés aux services de médecine généraliste, de santé publique et aux services spécialisés dans la prise en charge de la précarité. L'étude de la prise en charge des

5 Loi ${ }^{0} 70-1318$ du 31 décembre 1970 portant réforme hospitalière. 
populations démunies à l'hôpital public nécessite donc en premier lieu d'identifier d'une part les lieux à l'intérieur de l'institution dans lesquels ils se rendent principalement et, d'autre part, les mécanismes menant à l'exclusion de certains groupes des systèmes de protection maladie et du système de soin. L'analyse de la fonction sociale à l'hôpital moderne montre enfin que des inégalités sociales de santé sont inhérentes au fonctionnement de l'hôpital public moderne.

Ainsi, de nombreux travaux sociologiques qui se sont intéressés au fonctionnement de l'hôpital ont montré que cette institution produit des disparités au désavantage des classes sociales les plus basses. On a également vu que certains services accueillent particulièrement les patients les plus modestes, écartés en amont des circuits de soins classique du système de santé. Que peut nous dire la sociologie de ces lieux spécialisés dans la prise en charge de la pauvreté à l'hôpital public ? C'est ce que nous verrons dans la suite de l'article en prenant l'exemple de la PASS.

\section{L'analyse du traitement de la question sociale en santé et du gouvernement des pauvres à l'exemple de la PASS}

La "question sociale" est une aporie fondamentale sur laquelle une société expérimente l'énigme de sa cohésion et tente de conjurer le risque de sa fracture. Elle est un défi qui interroge, remet en question la capacité d'une société [...] à exister comme un ensemble lié par des relations d'interdépendance. (Castel, 2007, p. 25)

En mettant en scène l'action de la société pour intégrer les personnes dans le domaine de la santé, les Permanences d'accès aux soins de santé (PASS) invitent à s'interroger sur les fonctions de cette action en termes de régulation de l'ordre social et de gouvernement des populations qu'elles accueillent.

L'étude de la question sociale contemporaine met en miroir l'exclusion sociale avec une autre notion apparue dans les années 1980, celle de précarité. Le diagnostic établi de cette nouvelle «question sociale » lie la précarité et l'exclusion sociale à des risques et de l'insécurité. Il constitue depuis lors la manière principale de lire la « fracture sociale » (Gauchet, 2005) qui menace la cohésion de la société depuis la crise économique structurelle qui s'est installée après les chocs pétroliers de 1973 et 1979 et la fin des Trente Glorieuses et remet en cause la croyance dans la marche vers l'égalisation des conditions promise par l'essor de l'État providence.

La précarité est comprise comme «l'absence d'une ou plusieurs des sécurités, notamment celle de l'emploi, permettant aux personnes et familles d'assumer leurs obligations professionnelles, familiales et sociales, et de jouir de leurs droits fondamentaux» (Wresinski, 1987, p. 6). Dépassant une compréhension du phénomène de pauvreté comme exclusivement monétaire et comme un état figé dans le temps, la précarité est vue comme un processus dynamique et elle intègre toutes les dimensions de la vie sociale : le logement, la famille, le travail, la santé etc. La précarisation des différents domaines de la vie conduit à la désaffiliation et à l'exclusion sociale.

Les politiques d'assurance, visant à protéger contre certains risques contre une cotisation, ont largement dominé l'action de l'Etat social au $\mathrm{xx}^{\mathrm{e}}$ sicèle jusqu'aux années 1980. Adossée au travail salarier, l'efficacité de l'assurance a été remise en question avec l'arrivée d'un chômage endémique dans les années 1980. Les politiques d'assistance ont alors été remises au goût du jour. Elles sont le mode de gouvernement des pauvres qui domine dans les politiques publiques visant à « lutter contre l'exclusion » depuis les années 1980. Elle vise à apporter un secours ou une aide (sous forme de prestations monétaires, de biens ou de services) afin de protéger dans un domaine de la vie des individus ne disposant pas de ces protections et d'enrayer un processus d'exclusion. La permanence d'accès aux soins de santé s'inscrit directement dans le mouvement de renforcement de ces politiques assistancielles visant des groupes de la population menacés d'exclusion.

\section{La PASS : une structure assistancielle}

La PASS est une structure assistancielle archétypique. Elle fournit une aide ponctuelle qui 
ne peut être obtenue que sous certaines conditions (de pauvreté et de maladie) et après un examen individuel de la situation médicale et sociale par le médecin et/ou le travailleur social. L'aide est donc distribuée au cas par cas, instaurant une dimension de contrôle et permettant un travail fin de traduction des catégories administratives et d'assignation d'identités (Dubois, 2010).

La PASS fonctionne avec un système de bons organisé localement qui marque les limites de lieu et de temps : l'aide n'est disponible que dans l'hôpital où le bon est délivré (limitant la liberté du patient et son pouvoir de négociation) et seulement sur un temps limité (soit à un acte, soit une période de temps, en général un mois).

D'autre part, l'aide ne peut excéder le temps de la maladie ; on ne peut en effet théoriquement obtenir les services de la PASS si on est en bonne santé. Le système de bons souligne également le caractère exceptionnel et limité de l'aide (elle ne peut pas être distribuée à tous).

Son fonctionnement implique qu'un choix s'opère entre les bénéficiaires : certains seront intégrés au dispositif (et recevront une prise en charge médicosociale et des médicaments) et d'autres en seront exclus. Cette dernière caractéristique introduit une fonction de tri dans l'accès au dispositif entre des patients légitimes et d'autres illégitimes. ${ }^{6}$

La PASS, en tant que structure d'assistance, est une passerelle vers le système de droit commun. Elle vise à insérer ses patients dans le système de santé duquel ils sont éloignés. Cette assertion sépare d'emblée la PASS du droit commun, c'est-à-dire du système de santé ordinaire. Elle est pensée comme une porte d'entrée qui pallie temporairement et de manière exceptionnelle une absence d'accès aux soins de santé. L'insertion des patients dans le système de santé régulier est donc la condition sine qua non de l'activité de la PASS. Pourtant, comme on l'a vu dans la section précédente, les causes de l'exclusion du système de santé normal sont structurelles (exclusion de certaines catégories des mécanismes de protection maladie, réduction de la fonction sociale de l'hôpital, réformes des systèmes de protection sociale au désavantage des plus démunis etc.). Cela a pour conséquence de freiner la mission d'insertion de la PASS qui ne fonctionne pas toujours.

\section{Le gouvernement par l'assistance}

De nombreux travaux en sciences sociales ont montré les caractéristiques du mode de gouvernement de l'assistance et les effets contraires à ceux affichés en termes d'intégration et de cohésion sociales qu'il produit. Ainsi, les processus « d'individualisation du droit et des protections » que signent le renouveau des politiques d'assistance, identifiés par Robert Castel, tendent à responsabiliser les désaffiliés, plutôt que de remettre en cause les mouvements structuraux ayant mené à leur exclusion (Castel, 2007, p. 766). En privilégiant des critères soumis à appréciation, plutôt que des critères de droit, et des contreparties en termes « d'insertion » (recherche d'emploi, démarches administratives etc.), ces prestations introduisent une notion de mérite dans leurs mécanismes d'attribution et distinguent les pauvres méritants de ceux qui ne le sont pas (et par extension les «pauvres légitimes à recevoir l'aide » des «pauvres illégitimes »). En cela, l'assistance moderne moralise les pauvres et leur « assigne des identités » (qui s'inscrivent dans un spectre allant du «bon pauvre » au «mauvais pauvre »). Elle entretient par ailleurs une suspicion visant à protéger ses prestations des « abus » exercés par les demandeurs illégitimes et maintient ainsi une domination sociale envers ces publics. Enfin, Castel identifie des critères d'inclusion propres aux dispositifs d'assistance. Parmi eux, nous retiendrons celui de l'appartenance communautaire ou critère de proximité. Les structures protectrices dispensant l'aide s'occupent ainsi en priorité des personnes faisant partie de leur communauté (ainsi de nombreux dispositifs d'assistance ne s'adressent qu'aux personnes pouvant démontrer leur appartenance à une commune, une région, ou comme à la PASS, à un pays) (Castel, 2007, p. 62 et suiv.).

Vincent Dubois, de son côté, a insisté sur le rôle essentiel des street-level bureaucrats (les agents de ces administrations directement au contact

6 Caroline Izambert a montré que le tri des patients précaires à l’hôpital n'est pas seulement le fait des PASS, mais se déploie aussi dans les autres services hospitaliers (Izambert, 2016). 
du public) dans la réalisation de ces politiques, ils y jouent celui d' " entrepreneurs de morale ». Ils disposent d'une marge de manœuvre importante dans l'interprétation des règles leur donnant un pouvoir discrétionnaire et rendant l'attribution de l'aide incertaine (Dubois, 2010). L'assistance moderne renoue avec une tradition ancienne de l'assistance en contrôlant les comportements et en inculquant des normes de bonne conduite. Les différentes études ethnographiques font ressortir le rôle essentiel que jouent les ensembles de valeurs, de sentiments et d'émotions dans ce gouvernement par le bas laissant entrevoir la centralité d'une "économie morale »7 dans le domaine de l'assistance. Elle contient une tension fondamentale et historiquement ancrée entre une fonction d'aide et d'intégration d'un côté et une fonction de contrôle et de répression de l'autre, entre inclusion et exclusion (Castel, 2007; Foucault, 2009; Foucault et al., 1979; Geremek, 2010). Loin d'entrer en contradiction avec les réformes gestionnaires et les restrictions budgétaires que connaissent les différentes administrations publiques de l'État social, l'assistance s'adapte dans sa forme moderne aux règles du new public management, voire se redéfinit en fonction de ces dernières (Gabarro, 2012; Martin, 2016; Siblot, 2005).

Les études abordant cette question dans le domaine de la santé sont rares. Daniel Cefaï et Edouard Gardella se sont intéressés au dispositif d'assistance sociomédicale du Samusocial de Paris pour décrire «l'urgence sociale en action » et comprendre la mise en œuvre de cette politique sociale publique sur le terrain (Cefaï; Gardella, 2011). Grâce à une « ethnographie morale », ils décrivent notamment l'urgence comme une technique de gouvernement des sans-abri et en restituent ses dimensions politiques et morales. Isabelle Parizot, elle, a mené une enquête analysant l'assistance sociomédicale aux plus démunis dans deux types de centres de soins gratuits : les Permanences d'accès aux soins de santé (PASS) et les dispensaires associatifs de Médecins du Monde (Parizot,
2003). À l'aide d'une classification de différentes " carrières morales des assistés ", ${ }^{8}$ elle a étudié les rapports sociaux autour des processus identitaires et décrit les expériences vécues qu'engendre la fréquentation de ces lieux: «Les expériences vécues par les patients apparaissent intimement liées à leur capacité à faire face au discrédit de l'assistance. L'assistance sociomédicale représente en effet une expérience stigmatisée, conduisant en cela à une trajectoire institutionnelle particulière » (Parizot, 2003, p. 251-252).

L'auteur conclut son étude en suggérant que si l'assistance (dans le milieu associatif et hospitalier) discrédite et stigmatise ses usagers d'un côté, elle contribue à produire du lien social et à actualiser une solidarité de proximité de l'autre. Mais très peu d'éléments permettent de saisir l'autre face de l'assistance, celle qui reproduit les inégalités sociales de santé et contribue à la domination des groupes sociaux auxquels elle se destine en stratifiant le système de santé à différents niveaux. Les deux « univers symboliques » distincts de l'assistance en santé qu'elle décrit, l'un « médical » (ayant cours à l'hôpital) et l'autre "humaniste » (ayant cours dans le monde associatif) ne prennent pas assez en considération les divisions à l'intérieur de l'espace hospitalier ou d'autres facteurs (notamment organisationnels et budgétaires, mais pas seulement), pourtant essentiels pour comprendre les normes et les valeurs qui guident l'action dans les PASS.

L'enquête empirique que nous avons menée dans les PASS a permis d'identifier certaines caractéristiques du gouvernement assistanciel dans le domaine de la santé. Tout d'abord, il s'organise autour d'une forte tension entre inclusion et exclusion. Les critères d'éligibilité des patients pour intégrer le dispositif de la PASS sont évalués à la fois par la direction de l'hôpital et par les professionnels travaillant dans la structure (médecins et travailleurs sociaux). Le critère de proximité à la société française y joue un rôle central. Il est tout d'abord mesuré par le titre de séjour (ou son absence) et les droits sociaux qui y

\footnotetext{
7 Pour Didier Fassin, une « économie morale correspond à la production, la répartition, la circulation et l'utilisation des émotions et des valeurs, des normes et des obligations dans l'espace social » (Fassin; Eideliman, 2012, p. 12).

8 Isabelle Parizot emprunte cette notion à Serge Paugam (2009) qu'il utilise dans son analyse de la nouvelle pauvreté.
} 
sont attachés. Plus le statut de séjour est identifié comme révélateur d'une absence (de volonté) d'intégration à la société française (comme par exemple un visa de tourisme), plus les patients seront identifiés comme des "mauvais migrants" venus " profiter » de la gratuité du système de santé français. Les contours de l'assistance dans le domaine de la santé sont ainsi intimement liés à la définition de la citoyenneté, devenue l'étalon d'une forme de droit à l'intégration en santé porté par l'État, ses hôpitaux et son système d'assurance maladie (voir notamment Carde, 20o9; Izambert, 2010; Geeraert, 2017a). Paradoxalement, à la PASS ce n'est pas l'absence de statut légal qui empêche l'accès à ce droit - au contraire, il en ouvre plutôt l'accès car il est identifié par les professionnels comme appartenant à la catégorie des « migrants sans papiers vivant en France » (et donc répondant au critère de proximité de l'assistance) -, mais plutôt certaines formes de citoyenneté instables qui témoignent d'une précarité administrative importante (Geeraert, 2016). Ainsi, l'étude de la dimension assistancielle de la PASS dévoile que le traitement de la question sociale a aussi pour objet une certaine régulation du flux d'étrangers liée aux questions de santé. La PASS est l'outil d'une gouvernementalité pragmatique qui permet à la fois le contrôle et le maintien en bonne santé de certaines catégories de la population situées à la marge et en dehors de la citoyenneté et de la société (mais présentes sur le territoire). Elle contient ainsi les tensions sociales potentielles qu'engendreraient une exclusion complète du système de santé et crée par la même occasion une certaine subordination de ces patients envers la société française.

Le degré d'ouverture des critères d'inclusion et d'exclusion aux marges de l'assistance dépend des dispositions morales des professionnels. Celles-ci se construisent individuellement en fonction de l'habitus et des trajectoires sociales. De manière assez proche avec ce qu'a analysé Alexis Spire (2008) dans les préfectures, les professionnels des PASS voient dans leur travail une fonction de maintien de l'ordre à l'intérieur du système de protection sociale : protéger ce dernier contre les fraudes, les abus et reconnaître les patients méritants l'aide. ${ }^{9}$

\section{De l'augmentation du magistère de la médecine au gouvernement des pauvres}

L'étude du gouvernement des pauvres à l'hôpital comme il a lieu à la PASS invite à identifier ce que Didier Fassin a qualifié de "sanitarisation du social ". Ce phénomène récent désigne une traduction d'un problème social en phénomène sanitaire « où le politique se dit dans le langage médical » ou, en d'autres termes, une pathologisation et une médicalisation des problèmes sociaux (Fassin, 1998, p. 10, voir aussi Fassin, 1996). Ce concept est un développement des travaux rendant compte de la médicalisation de la société, c'est-à-dire de l'extension du pouvoir de la médecine dans la société (Conrad, 1992; Illich, 1982; sur la médicalisation en France, voir Aïach; Delanoë, 1998; Panese; Barras, 2008).

Mais ce n'est pas tout ce que la perspective biopolitique apporte à notre analyse. Les théoriciens de la biopolitique qui ont suivi Michel Foucault ont mobilisé la distinction aristotélicienne entre la zoé - la vie biologique, physique qui est la même chez les animaux et l'homme - et la bios - la vie de l'homme augmentée de sa dimension politique, celle inscrite dans un espace social..$^{10}$ Cette distinction permet de conceptualiser différentes valeurs

9 Le gouvernement de la migration par l'assistance n'a jamais fait l'objet de travaux à notre connaissance. En cela, nous rapprochons notre analyse de travaux étudiant le gouvernement de la migration par le bas dans d'autres lieux. Alexis Spire (2008) a étudié les « guichets de l'immigration " (préfectures, direction du travail, ambassades françaises à l'étranger) et a montré l'inscription de l'action des agents de ces guichets dans le maintien de l'ordre national. Ce dernier se décline entre l'ordre public (lutte contre la fraude), ordre moral (prévenir les possibles «abus » d'étrangers qui menacent le modèle social) et l'ordre économique (« utilité » pour la communauté française). On retrouve les mêmes éléments chez les professionnels de la PASS.

10 La distinction entre zoé et bios a été mobilisée et conceptualisée dans la philosophie moderne par Hannah Arendt (1988) et reprise par Giorgio Agamben qui l'a appliquée au concept foucaldien de biopolitique pour expliquer son concept de la vie nue. G. Agamben explique que la modernité remet en cause la différence et l'écart entre zoé et bios, elles « entrent dans une zone d'indifférenciation irréductible » ce qui rendrait possible le totalitarisme (Agamben, 1997, p. 17). 
de la vie selon qu'on considère la zoé ou la bios, distinction qui irait d'une vie biologique - ou vie nue - à une vie augmentée par ses dimensions sociales et politiques. Didier Fassin propose de concevoir la biopolitique en tant que politique du " vivant » au sens utilisé par Georges Canguilhem (2009) (c'est-à-dire compris dans sa matérialité, reliant le biologique au vécu expérientiel) (Fassin, 2006). Grâce à cet élargissement, D. Fassin a décrit la «raison humanitaire », ce mode de gouvernement de la question sociale basé sur des sentiments moraux comme la compassion, une échelle de légitimité de la souffrance et une protection de la vie dans sa dimension sacrée (Fassin, 2010). Ce dernier élément repose sur une conception d'un gouvernement biopolitique moral protégeant la zoé - ce qu'il appelle la bio-légitimité.

Miriam Ticktin, dans son ouvrage Casualties of care étudiant les politiques d'immigration française depuis la fin des années 1990, défend une idée similaire : l'emprise grandissante d'un mode de gouvernement de la migration sur un mode humanitaire (temporalité de l'urgence, centralité de la compassion et de la souffrance etc.). Elle montre notamment que la focalisation de l'exception humanitaire sur la souffrance tend à réduire l'aide apportée autour de la préservation de la vie biologique (zoê) au détriment d'une reconnaissance de la vie politiquement et socialement constituée (bios). La clause humanitaire permettant aux étrangers malades d'obtenir un titre de séjour temporaire, par exemple, ne permet pas d'obtenir automatiquement une autorisation de travailler. Ces étrangers malades obtiennent donc en quelque sorte un "droit de ne pas mourir ", sans que leur soient associés des droits politiques (Ticktin, 2011). En partant de ces résultats, nous nous sommes demandés dans quelle mesure la PASS participe à un «gouvernement humanitaire » qui s'inscrit dans un univers moral protégeant en priorité la vie biologique au détriment de la vie des patients augmentée de ses dimensions politiques et sociales.

La raison humanitaire, introduisant une politique compassionnelle qui valorise l'innocence et la souffrance, prend le relai du critère de proximité évoqué plus haut installant un régime d'exception aux extrémités de la solidarité assistancielle à la
PASS. Ainsi, l'inclusion des patients ne répondant pas aux critères classiques de l'assistance et n'étant donc pas d'emblée légitimes pour être pris en charge dans la PASS (comme les patients ne répondant pas au critère de proximité par exemple) va dépendre de la compassion que leur récit va susciter chez les professionnels de la PASS lors des premières situations de face-à-face. Moins le récit suscitera la compassion (parce que le professionnel n'y reconnaîtra pas de souffrance légitime), moins le patient aura de chances d'obtenir des soins. Ce régime d'exception guidé par l'émotion et la morale produit de la différence entre les migrants, s'opposant ainsi à une égalité de traitement. Une économie morale se substitue à l'économie politique lorsque cette dernière fait défaut afin de justifier l'exclusion de certains patients par la condamnation morale (catégorisés comme mauvais pauvres ou comme mauvais migrants, voir Geeraert, 2016) et d'en masquer les aspects politiques et historiques.

\section{Des valeurs de la vie à géométrie variable}

L'enquête empirique dans les PASS a montré une autre dimension liée directement à la conception de la vie attribuée aux patients par le système hospitalier. Deux cas de figure illustrent les ressorts du gouvernement biopolitique qui a lieu à la PASS. Le premier est celui où la PASS échoue dans son rôle d'insertion, c'est-à-dire où elle n'est plus une passerelle, mais la seule structure publique d'accès aux soins. Le second est celui de l'échec du rôle d'inclusion au sens fort du terme, c'est-à-dire lorsque la PASS - dernier filet de sécurité public - exclue définitivement le patient. Ces deux cas de figure correspondent à deux niveaux de stratification, le premier est celui d'une intégration limitée ou «bridée» (Chauvin, 2009) et le second, plus radical, correspond à l'exclusion ou au bannissement (lorsque la zoé, la vie biologique, n'est plus protégée).

Lorsque la mission d'insertion ne fonctionne pas et que les patients sont coincés dans le système de la PASS, cette dernière consolide alors un système de santé stratifié où elle représenterait un des derniers étages réservés aux moins désirables. La PASS devient une structure parallèle au droit commun qui tente de le remplacer avec peu de moyens et incarne 
une deuxième vitesse du système de santé pour des personnes exclues par la société.

La PASS est un espace assez singulier à l'hôpital. C'est un endroit où les pratiques de soin habituelles n'ont pas cours. Les patients y connaissent un traitement différencié dans leur prise en charge par rapport aux patients habituels de l'hôpital. Certes, ils peuvent y obtenir une prise en charge adaptée à leur situation sociale et culturelle (accompagnement social, service d'interprétariat etc.). Mais, au-delà de cette discrimination positive, les patients sans couverture maladie de la PASS connaissent un rationnement des soins : les différents examens (biologiques, radiologiques etc.) et les médicaments sont distribués au compte-goutte, souvent différés dans le temps lorsque cela est possible. À la PASS, le patient ne peut recevoir que les soins disponibles sur l'hôpital. La liste des médicaments disponibles pour les patients de la PASS est restreinte et exclut tous les médicaments jugés « de confort » ou dont l'efficacité médicale n'a pas été reconnue par les autorités sanitaires.

Ainsi, si l'accès à la médecine générale, au service social et aux médicaments de base et peu chers ne sont pas remis en cause, tous les autres soins sont susceptibles de l'être, surtout lorsque leur coût est élevé. La première catégorie de soins, celle qui n'est pas remise en cause, est légitime car elle bénéficie d'un mode de financement qui en invisibilise les coûts ou concerne des biens dont le coût est négligeable. L'illégitimité de la deuxième catégorie (tous les autres soins) est causée par une mise en exergue de ses coûts absolus : on expose le prix des soins qui doivent être portés par l'hôpital (alors que chez les patients assurés ces coûts sont en grande partie invisibles et portés par l'assurance maladie). La forte exposition du coût des soins dans un contexte budgétaire contraint construit (artificiellement) l'impression que les patients des PASS coûtent chers, alors même qu'ils n'ont qu'un accès réduit aux soins.

Le rationnement des soins connaît certaines limites. Nous avons distingué deux principaux régimes d'exception. La PASS en tant que système d'assistance contient tout d'abord une dimension « humanitaire ». ${ }^{11}$ Elle peut être résumée par le titre de l'article d'Estelle Carde (2006) «On ne laisse mourir personne ", phrase qui revient comme un leitmotiv dans la bouche de ses enquêtés pour signifier les limites aux discriminations qu'exercent ces professionnels de l'accès aux soins sur leurs patients. Cette limite au rationnement et à l'exclusion des soins se construit à l'aide d'une représentation de la vie nue et rend légitime les soins qui sont nécessaires à la préservation de la vie biologique des patients lorsque cette dernière est menacée. Une biolégitimité est donc reconnue aux patients de la PASS.

Une autre limite au rationnement réside dans des considérations de santé publique. La PASS participe à la mission historique de protection des grands fléaux et prend en charge tous les patients atteints de maladies infectieuses et contagieuses (par exemple tuberculose ou VIH), les limites de l'assistance et budgétaires importent peu dans ce cas-là. Une activité de prévention et de dépistage est également réalisée. Cette mission de santé publique est centrale à la PASS, elle montre une autre facette de la biopolitique en cours dans cette structure. Le traitement de la question sociale dans le domaine de la santé vise à protéger la société dans son ensemble contre les risques sanitaires que les « indésirables du système de santé » pourraient causer. La question sociale qui transparaît aussi à la PASS est celle du gouvernement et du contrôle biopolitiques de groupes dont l'exclusion du système de santé représente un potentiel danger sanitaire.

La PASS, dans cette dimension, devient un lieu d'apprentissage et d'intériorisation des différences fondamentales, ontologiques, de certains patients. On y voit se dessiner une catégorie de sous-patients chez qui on reconnaît une vie nue (cette vie biologique dénuée de ses dimensions politiques), celle dont la protection se mesure en termes de " nécessité vitale » ou "d'extrême gravité ». La valeur de la vie des patients de la PASS est moindre par rapport à celle des patients intégrés au système de santé principal.

\footnotetext{
11 Dans le sens que Didier Fassin (2010) donne à ce terme, c'est-à-dire une action conduite par un impératif moral guidé par la compassion et la reconnaissance de la souffrance et du malheur chez certaines personnes.
} 


\section{Conclusion}

Le traitement de la question sociale en santé s'enracine dans une histoire plurielle qui est loin de se résumer à une protection de la santé des plus démunis. La prise en charge des plus pauvres par l'hôpital public en France est intimement liée à la question de l'organisation sociale des sociétés complexes et de leur régulation. L'hôpital constitue historiquement une solution pour intégrer les groupes sociaux menacés d'exclusion dans une société différenciée. Mais cette fonction régulatrice ne s'exprime pas seulement par la solidarité ; elle contient une tension fondamentale entre inclusion et exclusion, entre contrôle et répression d'un côté et intégration et devoir de secours de l'autre.

La prise en charge de la pauvreté à l'hôpital public sur la période récente est façonnée par les transformations qui ont lieu à l'intérieur de l'institution (restrictions budgétaires, spécialisation, réduction de la fonction sociale) et dans le système de protection maladie (ayant conduit à sa stratification pour des catégories d'étrangers précaires). L'analyse de cet objet - à l'exemple de la Permanence d'accès aux soins de santé - sous l'angle du gouvernement assistanciel et de la biopolitique permet de dégager plusieurs caractéristiques du traitement de la question sociale tel qu'il à lieu à l'hôpital public français.

On peut dégager trois grandes fonctions du gouvernement à la PASS. Tout d'abord, une fonction humanitaire, celle de protéger la vie nue de ces groupes dominés leur assurant une bio-légitimité. Ensuite, une fonction de tri, caractérisée par l'inclusion et l'exclusion et qui vise à séparer les migrants légitimes et méritants des migrants illégitimes. À cette activité, s'ajoute celle d'insertion des migrants légitimes dans le système de santé, permettant d'assurer le maintien en bonne santé de catégories menacées d'exclusion sociale. Enfin, une mission de santé publique qui consiste à prendre en charge les patients atteints de maladies infectieuses et transmissibles. Toutes ces fonctions sont traversées par des préoccupations budgétaires qui structurent les modes de prise en charge s'inscrivant dans un mouvement plus général d'un tournant néomanagérial.
Cette biopolitique de la question sociale se construit autour d'un bricolage compliqué qui mêle citoyenneté, politiques économiques, politiques de santé publique et accès aux soins. Le mode d'exercice du pouvoir se caractérise par un gouvernement par le bas qui est réalisé par les professionnels de terrain lors des interactions avec les patients. Il met en scène une économie morale basée sur des représentations de ce qui est bon et mauvais pour la société.

\section{Références}

AGAMBEN, G. Homo sacer: le pouvoir souverain et la vie nue. Paris: Seuil, 1997.

AÏACH, P.; DELANOË, D. (Org.). L'ère de la

médicalisation. Paris: Economica, 1998.

ARENDT, H. Condition de l'homme moderne. Paris: Pocket, 1988.

BELORGEY, N. L'hôpital sous pression. Paris: La Découverte, 2010.

BENAMOUZIG, D. La santé au miroir de

l'économie: une histoire de l'économie de la santé en France. Paris: PUF, 2005.

BEZES, P. État, experts et savoirs néomanagériaux: les producteurs et diffuseurs du New Public Management en France depuis les années 1970. Actes de la Recherche en Sciences Sociales, Paris, v. 193, n. 3, p. 16-37, 2012.

BORGETTO, M. Santé et action sociale: d'hier à aujourd'hui. Les Tribunes de la Santé, Paris, v. 38 , n. 1, p. 29-40, 2013.

CANGUILHEM, G. Le normal et le pathologique. Paris: PUF, 2009.

CARDE, E. "On ne laisse mourir personne": les discriminations dans l'accès aux soins, Travailler, Paris, n. 2, p. 57-8o, 2006.

CARDE, E. Quinze ans de réforme de l'accès à une couverture maladie des sans-papiers: de l'Aide sociale aux politiques d'immigration. Mouvements, Paris, v. 59, n. 3, p. 143-156, 2009.

CASTEL, R. Les métamorphoses de la question sociale: une chronique du salariat. Paris: Gallimard, 2007. 
CASTEL, R. La montée des incertitudes: travail, protections, statut de l'individu. Paris: Seuil, 2009.

CEFAÏ, D.; GARDELLA, É. L'urgence sociale en action: ethnographie du Samu social de Paris. Paris: la Découverte, 2011.

CHAUVENET, A. Idéologies et statuts professionnels chez les médecins hospitaliers. Revue Française de Sociologie, Paris, v. 14, n. 1, p. 61-76, 1973 .

CHAUVIN, S. En attendant les papiers: l'affiliation bridée des migrants irréguliers aux États-Unis. Politix, Paris, v. 87, n. 3, p. 47-69, 2009.

COLOMBO, F.; MORGAN, D. Évolution des dépenses de santé dans les pays de l'OCDE. Revue Française des Affaires Sociales, Paris, n. 2-3, p. 1942, 2006.

CONRAD, P. Deviance and medicalization: from badness to sickness. Philadelphia: Temple University Press, 1992.

DODIER, N.; CAMUS, A. L'hospitalité de l'hôpital. Communications, Lyon, v. 65, n. 1, p. 109-119, 1997.

DODIER, N.; CAMUS, A. Openness and specialisation: dealing with patients in a hospital emergency service. Sociology of Health and Illness, Hoboken, v. 20, n. 4, p. 413-444, 1998.

DOMIN, J.-P. La nouvelle gouvernance ou le couronnement de vingt ans de réformes hospitalières. Économies et Sociétés, Paris, n. 8, p. $459-487,2007$.

DUBOIS, V. La vie au guichet: relation administrative et traitement de la misère. Paris: Economica, 2010.

FASSIN, D. L'espace politique de la santé: essai de généalogie. Paris: Presses Universitaires de France, 1996.

FASSIN, D. Les politiques de la médicalisation. In: AÏACH, P.; DELANOË, D. L'ère de la médicalisation. Paris: Economica, 1998. p. 1-13.

FASSIN, D. La biopolitique n'est pas une politique de la vie. Sociologie et Sociétés, Montreal, v. 38, n. 2, p. 35-48, 2006.
FASSIN, D. La raison humanitaire: une histoire morale du temps présent. Paris: Gallimard: Seuil, 2010.

FASSIN, D.; COSTA-LASCOUX, J.; HILY, M.-A. L'altérité de l'épidémie: les politiques du sida à l'épreuve de l'immigration. Revue Européenne de Migrations Internationales, Poitiers, v. 17, n. 2, p. 139-151, 2001.

FASSIN, D.; EIDELIMAN, J.-S. Introduction: defense et illustrations des économies morales. In: FASSIN, D.; EIDELIMAN, J.-S. (Dir.). Economies morales contemporaines. Paris: La Découverte, 2012. p. 9-18.

FASSIN, D.; MEMMI, D. (Eds.). Le gouvernement des corps. Paris: EHESS, 2004.

FOSSIER, A. Le grand renfermement. Tracés: Revue de Sciences Humaines, Lyon, n. 1, 2002.

FOUCAULT M. Histoire de la folie à l'âge classique. Paris: Gallimard, 1976a.

FOUCAULT, M. Histoire de la sexualité I: la volonté de savoir. Paris: Gallimard, 1976b.

FOUCAULT, M. Naissance de la biopolitique: cours au Collège de France (1978-1979). Paris: Ehess, 2004.

FOUCAULT, M. Naissance de la clinique. Paris: Presses Universitaires de France, 2009.

FOUCAULT, M. et al. Les machines à guérir: aux origines de l'hôpital moderne. Bruxelles: P. Mardaga, 1979.

GABARRO, C. Les demandeurs de l'aide médicale d'État pris entre productivisme et gestion spécifique. Revue Européenne des Migrations Internationales, Poitiers, v. 28, n. 2, p. 35-56, 2012.

GAUCHET, M. Le désenchantement du monde: une histoire politique de la religion. Paris: Gallimard, 2005 .

GEERAERT, J. Le touriste et le réfugié. Cliniques Méditerranéennes, Toulouse, n. 94, p. 69-82, 2016.

GEERAERT, J. Healthcare reforms and the creation of ex-/included categories of patients: "irregular migrants" and the "undesirable" in the French Healthcare System. International Migration, Hoboken, v. 56, n. 2, p. 68-81, 2017 a. 
GEERAERT, J. La question sociale en santé:

l'hôpital public et l'accès aux soins des personnes en marge du système de santé en France à l'aube du XXIe siècle. 2017. Tese (Doutorado em Sociologia) Université Paris 13, Villetaneuse, 2017b.

GEREMEK, B. La potence ou la pitié: l'Europe et les pauvres du Moyen Âge à nos jours. Paris: Gallimard, 2010.

GORI, R.; DEL VOLGO, M.-J. La santé totalitaire: essai sur la médicalisation de l'existence. Paris: Flammarion, 2009.

HERZLICH, C. Types de clientèle et fonctionnement de l'institution hospitalière. Revue Française de Sociologie, Paris, v. 14, n. 1, p. 41-59, 1973.

ILLICH, I. Medical nemesis: the expropriation of health. New York: Pantheon Books, 1982.

IMBERT, J. Les hôpitaux en France. Paris: Presses Universitaires de France, 1966.

IMBERT, J.; MOLLAT, M. Histoire des hôpitaux en France. Toulouse: Privat, 1982.

IZAMBERT, C. 30 ans de régressions dans l'accès aux soins. Plein Droit, Paris, v. 3, n. 86, p. 5-9, 2010.

IZAMBERT, C. Logiques de tri et discriminations à l'hôpital public: vers une nouvelle morale hospitalière? Agone, Marseille, n. 58, p. 89-104, 2016.

JAMOUS, H. Sociologie de la décision: la réforme des études médicales et des structures hospitalières. Paris: CNRS, 1969.

LE GARREC, M.-A.; KOUBI, M.; FENINA, A. 6o années de dépenses de santé: une rétropolation des Comptes de la santé de 1950 à 2010 . Études et Résultats, Paris, n. 831, p. 1-8, 2013.

MARTIN, P. Les métamorphoses de l'assurance maladie: conversion managériale et nouveau gouvernement des pauvres. Rennes: PUR, 2016.

OMS - ORGANISATION MONDIALE DE LA SANTÉ. Actes officiels de l'Organisation mondiale de la Santé. New York, 1946. n. 2.
PANESE, F.; BARRAS, V. Médicalisation de la "vie" et reconfigurations médicales. Revue des Sciences Sociales, Strasbourg, n. 39, p. 20-29, 2008.

PARIZOT, I. Soigner les exclus: identités et rapports sociaux dans les centres de soins gratuits. Paris: Presses Universitaires de France, 2003.

PAUGAM, S. La disqualification sociale: essai sur la nouvelle pauvreté. Paris: PUF, 2009.

PIERRU, F. Hippocrate malade de ses réformes. Bellecombe-en-Bauges: Croquant, 2007.

SIBLOT, Y. "Adapter" les services publics aux habitants des "quartiers difficiles": diagnostics misérabilistes et réformes libérales. Actes de la Recherche en Sciences Sociales, Paris, v. 159, n. 4, p. 70-87, 2005 .

SIBLOT, Y. Faire valoir ses droits au quotidien: les services publics dans les quartiers populaires. Paris: Presses de Science, 2006.

SICARD, D.; LE PEN, C. Santé: l'heure des choix. Paris: Desclée de Brouwer, 2004.

SPIRE, A. Accueillir ou reconduire: enquête sur les guichets de l'immigration. Paris: Raisons d'Agir, 2008.

STEUDLER, F. Hôpital, profession médicale et politique hospitalière. Revue Française de Sociologie, Paris, v. 14, n. 1, p. 13-40, 1973.

TICKTIN, M. Casualties of care: immigration and the politics of humanitarianism in France. Berkeley: University of California Press, 2011.

VASSY, C.; RENARD, F. Quels malades pour l'hôpital public? Deux stratégies de segmentation, Gérer et Comprendre, Paris, n. 27, p. 29-39, 1992.

WRESINSKI, J. Grande pauvreté et précarité économique et sociale. Paris: Conseil Économique et Social, 1987.

Reçu: 07/06/2018

Approuvé: 30/06/2018 